\title{
O AROMA AMBIENTAL E SUA RELAÇÃO COM AS AVALIAÇÕES E INTENÇÕES DO CONSUMIDOR NO VAREJO
}

\author{
AMBIENT SCENT AND ITS RELATIONSHIP WITH CONSUMER EVALUATIONS AND \\ INTENTIONS IN RETAIL \\ EL AROMA AMBIENTAL Y SU RELACIÓN CON LAS EVALUACIONES E INTENCIONES DEL CONSUMIDOR EN EL \\ COMERCIO MINORISTA
}

\section{RESUMO}

A atmosfera de uma loja é capaz de provocar emoções e comportamentos que estimulam a compra. Entre as suas dimensões, está o aroma ambiental, adicionado artificialmente ao ambiente. Este artigo investiga a relação entre a presença de aroma ambiental no varejo e as avaliações de loja, ambiente de loja e produtos, além das intenções comportamentais relativas ao retorno e ao tempo gasto na loja. A pesquisa teve uma etapa exploratória, exame de publicações em fisiologia, psicologia e comportamento do consumidor, para construção do referencial teórico e formulação das hipóteses, e outra causal, quase experimento, para identificar as relações de causa e efeito na presença de aroma. O estudo foi conduzido em uma butique de frutos do mar, e os instrumentos de coleta de dados, escalas de diferencial semântico, basearam-se em pesquisas similares. As análises incluíram técnicas de estatística descritiva e teste de hipóteses. Os resultados revelaram que a presença de aroma ambiental não aumentou as avaliações nem a intenção de retorno, mas reteve o consumidor por mais tempo na loja.

PALAVRAS-CHAVE Varejo, atmosfera de loja, aroma, olfato, comportamento do consumidor.

André Luiz Carvalho Nunes da Costa andrelcncosta@yahoo.com.br

Analista do Banco Central do Brasil - Brasília - DF, Brasil

Salomão Alencar de Farias saf@ufpe.br

Professor do Programa de Pós-Graduação em Administração da Universidade Federal de Pernambuco - Recife - PE, Brasil

\begin{abstract}
The atmosphere of a store can be used to produce specific emotions that enhance purchase probability, and the ambient scent, artificially added, is among its dimensions. This article investigates the relationship between the presence of an ambient scent in a retail store, and customers' evaluation of the store, its environment and products, as well as the behavioral intentions related to return and time spent in the store. The research design included an exploratory stage, examination of publications in the fields of physiology, psychology and consumer behavior, to provide the conceptual background and to formulate research hypothesis; and a causal stage, quasi-experiment, to verify the effects of scent presence. Semantic differential scales were employed for data collection. Descriptive statistics and hypothesis test were performed for data analysis and the results revealed that the scent presence did not improve the evaluations or the intent to return but retained the consumer longer in the store.

keywords Retail, store atmosphere, scent, olfaction, consumer behavior.

Resumen La atmósfera de un negocio es capaz de generar emociones y comportamientos que estimulan la compra. Entre sus dimensiones, está el aroma ambiental, adicionado de modo artificial al ambiente. Este artículo averigua la relación entre la presencia de aroma ambiental en el comercio minorista y las evaluaciones de negocio, ambiente de negocio y productos, además de las intenciones de comportamiento con respecto al retorno y al tiempo dedicado al negocio. La investigación tuvo una etapa exploratoria, examen de publicaciones en fisiología, psicología y comportamiento del consumidor, para construcción del referencial teórico y formulación de las hipótesis, y otra causal, casi-experimento, para identificar las relaciones de causa y efecto en la presencia del aroma. El estudio fue conducido en una especie de boutique para frutos del mar y, los instrumentos de colecta de datos, escalas de diferencial semántico, basaranse en investigaciones similares. Los análisis han incluido técnicas de estadística descriptiva y teste de hipótesis. Los resultados han revelado que la presencia de aroma ambiental no aumentó las evaluaciones ni la intención del retorno, pero mantuvo el consumidor por más tiempo en el negocio.

Palabras clave Comercio minorista, atmósfera del negocio, aroma, olfato, comportamiento del consumidor.
\end{abstract}




\section{INTRODUÇÃO}

A influência do ambiente sobre o comportamento do consumidor há muito tem sido reconhecida por varejistas e arquitetos. Uma loja representa o ponto de culminância entre o marketing de uma organização e seus clientes (PARENTE, 2000; PAL e BYROM, 2003). Nela, várias compras são decididas, por vezes, em função de um ambiente bem-planejado que estimule o consumidor.

Em marketing, o ambiente de loja é denominado "atmosfera", que, segundo Kotler (1973), é planejada para causar efeitos emocionais nos clientes, alterando seu estado de humor e favorecendo a compra. Os estímulos físicos do ambiente, como os de uma loja, podem influenciar indivíduos mesmo que estes não tenham consciência da percepção (TURLEY e MILLIMAN, 2000; DAVIDOFF, 2001; DAVIES e outros, 2003) e são captados por meio dos sentidos da visão, audição, olfato e tato. Kotler (1973, p. 51) divide-os em dimensões visuais (cores, formas, tamanhos), sonoras (música), olfativas (aromas, odores) e tácteis (maciez, temperatura). Segundo Bitner (1992), indivíduos respondem às dimensões da atmosfera de maneira holística, ou seja, embora certos estímulos já possam individualmente afetar o consumidor, o conjunto influenciará a resposta.

As respostas emocionais aos estímulos ambientais podem ser, segundo o paradigma EOR (estímulo - organismo - resposta) de Mehrabian e Russell (1974), de três naturezas: prazer, ativação e dominância. A dimensão prazer refere-se ao quanto o ambiente é agradável ou desagradável. A ativação é um estado de sentimento que pode ser representado por uma escala que vai da sonolência à excitação extrema. A última dimensão, dominância, refere-se a respostas caracterizadas por um sentimento de submissão ou domínio em relação aos estímulos e ao ambiente.

No varejo, as respostas emocionais aos estímulos influenciam comportamentos de aproximação ou afastamento do consumidor, como a sua intenção de voltar ou não à loja, sua frequência de compras, tempo e dinheiro gastos e a sua interação com a força de vendas (DONOVAN e ROSSITER, 1982, p. 37). Dessa forma, cabe aos profissionais de varejo a eficiente gestão dos estímulos de sua atmosfera, para gerar comportamentos de aproximação dos consumidores.

A escolha das cores, por exemplo, é primordial em muitos tipos de atmosfera, como em restaurantes, onde são usadas para segmentar o público (STROEBELE e De CASTRO, 2004). A temperatura é um estímulo táctil que exerce papel discreto na atmosfera da loja, mas passará a ser notada negativamente ao tornar-se desagradável (WAKEFIELD e BAKER, 1998). A música vem sendo utilizada há anos nos mais diversos tipos de varejo. Sua presença pode influenciar o ritmo de compra dos consumidores (MILLIMAM, 1982), o julgamento de indivíduos presentes no ambiente (DUBÉ e MORIN, 2001) e a aproximação em relação aos vendedores (TURLEY e CHEBAT, 2002).

O estímulo olfativo vem sendo usado na composição da atmosfera de vários tipos de varejo (GULAS e BLOCH, 1995; MITCHELL e outros, 1995; SPANGENBERG e outros, 1996; DAVIES e outros, 2003; MILOTIC, 2003; SPANGENBERG e outros, 2006; GOLDKUHL e STYVÉN, 2007; PARSONS, 2009). Em alguns deles, como padarias e cafeterias, o aroma é natural, exalado pelos próprios produtos vendidos. Entretanto, o avanço da tecnologia na aromatização de ambientes tem tornado possível a empresas de diferentes segmentos, a utilização desse estímulo (SPANGENBERG e outros, 1996; CHEBAT e MICHON, 2003; SPANGENBERG e outros, 2005; BOSMANS, 2006).

Mas quais seriam as razões para se incorporar o estímulo olfativo na atmosfera de loja? Quais são as vantagens, as desvantagens e os pontos críticos na incorporação desse estímulo ao varejo? Este artigo examina os efeitos práticos da adição de aroma ambiental (não oriundo de produtos) a uma butique de frutos do mar, mais especificamente em relação a avaliações e comportamentos de aproximação do consumidor.

\section{O ESTÍMULO OLFATIVO COMO PARTE DA ATMOSFERA DE LOJA}

O olfato e o paladar são os chamados sentidos químicos e estão intimamente ligados, sendo sua principal função a de permitir aos animais a distinção entre substâncias benéficas e prejudiciais (DAVIDOFF, 2001, p. 148). Cheiros são substâncias químicas suspensas no ar que excitam receptores presentes no alto da cavidade nasal. Os cheiros são processados próximo aos centros da emoção (amygdala) e da memória (bippocampus), exigindo praticamente nenhum esforço cognitivo para serem sentidos e para recuperar emoções e memórias (EHRLICHMAN e HALPERN, 1988; HERZ e ENGEN, 1996; CORBETT; 2006). Por essa razão, o olfato é considerado o sentido mais li- 
gado às reações emocionais (RICHARDSON e ZUCCO, 1989; MICHON e outros, 2005). Outras funções do olfato são a estética e a de determinação do sabor de bebidas e alimentos (DOTY, 1997).

No varejo, o estímulo olfativo geralmente recebe a denominação de aroma e a sua influência pode, de acordo com Gulas e Bloch (1995), ser de dois tipos: o aroma específico de objetos e o aroma presente no ambiente. O primeiro refere-se a pistas utilizadas pelo consumidor para avaliar a qualidade e os atributos dos produtos (BONE e JANTRANIA, 1992), principalmente daqueles em que o cheiro é um fator primordial, como alimentos, bebidas, cosméticos e produtos de limpeza (MILOTIC, 2003).

Aromas ambientais são aqueles que estão presentes no ambiente, mas não são exalados por produtos (BOSMANS, 2006; PARSONS, 2009). Podem influenciar as reações a todos os produtos vendidos em uma loja, mesmo aqueles aos quais seria difícil ou inapropriado adicionar um cheiro artificial (GULAS e BLOCH, 1995). Para alguns pesquisadores (por exemplo, DONOVAN e ROSSITER, 1982; BOSMANS, 2006), o cheiro é primordialmente um componente enriquecedor da atmosfera de loja, cuja influência sobre o consumidor vai muito além da comunicação dos atributos ou qualidades dos produtos.

\section{Fatores moderadores do aroma ambiental no varejo}

Diversos fatores moderam as respostas afetivas do consumidor do varejo ao estímulo olfativo. Os principais são a sua qualidade afetiva e a sua congruência com elementos do ponto de venda (GULAS e BLOCH, 1995; DAVIES e outros, 2003; SCHINDLER e HOLBROOK, 2003).

A resposta afetiva a um estímulo está relacionada com a sua capacidade de provocar alterações no estado de humor de um indivíduo (EHRLICHMAN e HALPERN, 1988). O humor é definido como um estado de sentimento e desempenha relevante papel no comportamento do consumidor, por sua capacidade de favorecer a recuperação de memórias e aumentar o envolvimento com a compra (HOLBROOK e HIRSCHMAN, 1982; HIRSCHMAN e HOLBROOK, 1982; GARDNER, 1985; BITNER, 1992; SCHMITT, 1999).

Nesse sentido, aromas são úteis para alterar o humor dos consumidores até um estado desejado pelo varejista (MITCHELL e outros, 1995), uma vez que a natureza do processamento olfativo nos seres humanos, que acontece muito próximo aos centros da emoção e memória no cérebro, favorece as respostas afetivas (HERZ e ENGEN, 1996). Um estudo de Ehrlichman e Halpern (1988), por exemplo, verificou que cheiros agradáveis favorecem a recuperação de memórias felizes.

Pesquisas realizadas por Spangenberg e outros (1996), Chebat e Michon (2003) e McDonnell (2007) encontraram evidências de que aromas ambientais prazerosos são capazes de produzir comportamentos de aproximação no varejo, como elevações na intenção de retornar ao local e no tempo gasto na visita à loja. De fato, por meio de um estudo estatístico em que examinaram vários artigos sobre a influência do aroma no varejo, Bone e Ellen (1999) concluíram que as qualidades afetivas (prazer e desprazer) do cheiro estão positivamente relacionadas com um maior tempo gasto na loja e um aumento nas intenções de retorno, como previram Donovan e Rossiter (1982), ao aplicarem o paradigma EOR (MEHRABIAN e RUSSELL, 1974) ao varejo.

Essas evidências permitem a formulação das seguintes hipóteses de pesquisa: (H1) a presença de aroma ambiental, comparada à sua ausência, aumenta as intenções de retornar à loja e (H2) a presença de aroma ambiental, comparada à sua ausência, aumenta o tempo de permanência dos clientes na loja.

Além de detectar o aumento do tempo gasto por indivíduos em visitas a lojas aromatizadas, experimentos feitos por Spangenberg e outros (1996) e Spangenberg e outros (2006) evidenciaram que os membros do grupo experimental apresentaram percepção distorcida do tempo gasto na visita. Essa constatação abre possibilidade para a formulação de mais uma hipótese: (H3) a presença de aroma ambiental, comparada à sua ausência, tende a fazer com que os clientes apresentem uma distorção na percepção do tempo gasto na loja.

Além de estimularem comportamentos de aproximação, as alterações positivas no estado de humor do consumidor favorecem as avaliações de produto e loja (DONOVAN e ROSSITER, 1982; GARDNER, 1985; BITNER, 1992; GULAS e BLOCH, 1995; SPANGENBERG e outros, 1996; CHEBAT e MICHON, 2003). Como visto acima, aromas agradáveis são um poderoso estímulo no favorecimento de estados de humor positivos, sendo capazes, por consequência, de melhorar as avaliações de produtos e loja (CHEBAT e MICHON, 2003; McDONNELL, 2007).

Em um experimento em que foi simulada uma loja de artigos para universitários, Spangenberg e outros (1996) registraram significativa melhora nas avaliações 
do ambiente e dos produtos vendidos quando a loja foi aromatizada com uma fragrância agradável. Parsons (2009) realizou um experimento no qual imagens de lojas foram positivamente avaliadas na presença de aromas agradáveis. Entretanto, para estes e outros autores, como Bone e Jantrania (1992) e Bosmans (2006), não basta que um aroma ambiental seja considerado agradável para favorecer as avaliações de loja e produtos, sendo fundamental que o cheiro seja congruente, típico ou apropriado ao produto ou à loja para gerar avaliações positivas.

De acordo com Mitchell e outros (1995), aromas ambientais congruentes podem recuperar emoções e memórias ligadas à loja e ao produto vendido, favorecendo a compra, enquanto cheiros incongruentes podem resgatar emoções e memórias incompatíveis com a loja e os produtos, interferindo negativamente no contexto. Exemplificando, um aroma adocicado pode ser apropriado em uma confeitaria, mas totalmente contraproducente em uma churrascaria.

Na venda de produtos que não exalam cheiro naturalmente, existe a opção da congruência por associação, em que a aromatização da loja é feita segundo algum critério de congruência contextual ou temática (SCHIFFERSTEIN e BLOK, 2002; DAVIES e outros, 2003; GOLDKUHL e STYVÉN, 2007; PARSONS, 2009). Seguindo essa linha de raciocínio, Schifferstein e Blok (2002) aromatizaram a seção de revistas de futebol de uma livraria com aroma de grama.

Com base nas considerações sobre a influência do aroma ambiental nas avaliações de loja e produto, são formuladas as três últimas hipóteses desta pesquisa, a saber: (H4) a presença de aroma ambiental produz avaliações da loja que são mais positivas do que as produzidas em ambiente sem a presença de cheiro; (H5) a presença de aroma ambiental produz avaliações do ambiente da loja que são mais positivas do que as produzidas em ambiente sem a presença de cheiro; e (H6) a presença de aroma ambiental produz avaliações dos produtos ofertados na loja que são mais positivas do que as produzidas em ambiente sem a presença de cheiro.

\section{PROCEDIMENTOS METODOLÓGICOS}

A pesquisa aplicada neste artigo foi estabelecida em duas etapas, uma exploratória e uma causal. Na primeira fase, foi realizada a revisão de literatura para uma melhor compreensão e aprofundamento do tema, bem como para identificação de escalas para mensuração de aroma e de avaliações no ponto de venda. Desse modo, escolhidas as escalas, fez-se o seu pré-teste (MALHOTRA, 2001; SAMPIERI e outros, 2006). A segunda fase, causal (MALHOTRA, 2001), consistiu na realização de um quase experimento (SAMPIERI e outros, 2006) para se obterem evidências de relações de causa e efeito (MALHOTRA, 2001) entre os construtos investigados. O pré-teste do aroma e sua calibração foram realizados nessa fase. Os dados foram coletados primeiramente no grupo de controle e em seguida no grupo experimental, já com a loja aromatizada.

O modelo quase experimental adotado por esta pesquisa foi conduzido em um varejo que comercializa frutos do mar de alta qualidade e produtos ligados à sua gastronomia (vinhos e especiarias), localizado em centro comercial planejado, configurando-se, portanto, uma pesquisa de campo (SAMPIERI e outros, 2006). A loja foca um público bastante segmentado, caracterizado pelo alto grau de escolaridade e bom nível cultural. São homens e mulheres bem-sucedidos, pertencentes às classes $\mathrm{A}$ e $\mathrm{B}$, que cultivam hábitos sofisticados e vão até o local em busca de produtos para seus rituais gastronômicos ou para presentear alguém. A decoração temática do espaço (de cerca de $70 \mathrm{~m}^{2}$ ) mescla sofisticação e rusticidade, com o emprego de materiais nobres, como o vidro e o metal escovado, e rústicos, como a madeira e a palha de fibras naturais, bastante comuns em ambiente de praia. A cuidadosa exposição dos itens e as belas imagens de pratos finais nas paredes convidam à exploração do ambiente e à compra por impulso. Muitas pessoas entram no local por curiosidade ou para conferir as novidades. Uma sofisticada tecnologia de embalagem e conservação mantém o local absolutamente limpo e totalmente livre de cheiros. Essas características justificam a elegibilidade da loja para aromatização, um antigo desejo dos empreendedores para realçar a sua já bem-idealizada atmosfera.

A variável independente desta pesquisa foi o aroma ambiental, adicionado à loja durante a fase experimental do estudo. As variáveis dependentes foram baseadas nas utilizadas por Spangenberg e outros (1996), que pesquisaram a influência da presença de aroma ambiental na avaliação de uma loja simulada em laboratório, e incluem a avaliação da loja pelo consumidor; a avaliação do ambiente da loja pelo consumidor; a avaliação dos produtos ofertados na 
loja, pelo consumidor; e as intenções comportamentais do consumidor em relação a retorno e tempo de permanência no local, incluindo uma verificação da percepção do tempo da visita.

Os instrumentos de medição, escalas de diferencial semântico, obtidas na pesquisa de Spangenberg e outros (1996), originalmente em inglês, foram traduzidos pelo método da tradução reversa (MALHOTRA, 2001). Escalas são recomendadas para medir atitudes, como a avaliação de um objeto (SAMPIERI e outros, 2006). Nas duas primeiras avaliações, as escalas foram múltiplas, ou seja, combinaram diversas variáveis que mediram o mesmo conceito, medida composta, para aumentar a confiabilidade (HAIR e outros, 2005). A seguir, o detalhamento de cada medição:

- Avaliação da loja: escala de diferencial semântico de sete pontos, com quatro pares de adjetivos (Tabela 1). Os clientes foram orientados a julgar aspectos como localização, atendimento, imagem, qualidade e condições de compra. Além da escala, foi feita uma pergunta fechada, na qual os respondentes deveriam informar se gostaram ou não da loja, classificando em seguida a intensidade dessa atitude em uma escala de sete pontos, com rótulos bipolares baixa/alta. A multiplicação de uma pela outra gerou uma escala de 14 pontos revelando o grau de aprovação/desaprovação da loja. As perguntas foram: "Que avaliação o(a) senhor(a) faz desta loja?", "O(a) senhor(a) gostou desta loja?" e "Com que intensidade o(a) senhor(a) gostou ou não desta loja?"

- Avaliação do ambiente de loja: escala de diferencial semântico de sete pontos e 14 pares de adjetivos bipolares (Tabela 1). A pergunta foi: "Que avaliação o(a) senhor(a) faz do ambiente desta loja?"

- Avaliação dos produtos disponíveis na loja: escala diferencial semântico de sete pontos, baseada na escala desenvolvida por Bellizzi e outros (1983), para valorização de percepções sobre mercadorias. Foram avaliados três itens, cada um deles com um par de adjetivos bipolares (Tabela 1). A pergunta foi: "Que avaliação o(a) senhor(a) faz dos produtos vendidos nesta loja?"

- Avaliação da intenção de retorno à loja: escala de sete pontos, na qual os respondentes deveriam indicar a possibilidade de retornarem à loja, assumindo que fossem comprar futuramente aqueles produtos. A pergunta foi: "Assumindo que o(a) senhor(a) fosse comprar esse tipo de produto futuramente, que possibilidade haveria de voltar a esta loja?"

O tempo de permanência dos clientes na loja foi cronometrado a partir de sua entrada no local, para comparação com suas respostas aos serem solicitados a estimar, sem consultar relógio, o tempo da visita. Para os clientes que não efetuaram compras, o cronômetro foi parado no momento da saída loja. Para os que compraram, a contagem parou na chegada ao caixa. Apenas foram entrevistados os clientes cuja trajetória na loja foi acompanhada e cronometrada desde o seu ingresso no local. Os tempos coletados foram convertidos para segundos, sendo, em seguida, calculadas as médias dos grupos, conforme procedimento adotado por Spangenberg e outros (1996).

Todos os dados foram coletados por meio de questionário estruturado. Além das escalas para medição das variáveis dependentes, havia questões fechadas sobre gênero, idade, local de residência, estado civil, escolaridade, renda familiar mensal e frequências de visita e de compra na loja, para levantamento do perfil demográfico dos grupos. A estimativa do tempo gasto na visita foi coletada por pergunta aberta: "Sem olhar no relógio, quanto tempo o senhor imagina ter permanecido dentro desta loja?" As abordagens foram feitas pelo próprio pesquisador, assim como a cronometragem, registrada em campo específico no questionário.

Os questionários foram pré-testados em uma loja da mesma cadeia, para analisar o tempo necessário para aplicação, a compreensão dos respondentes a respeito das escalas e perguntas e a sua adequação para medir as variáveis dependentes de pesquisa. Foram feitos alguns ajustes nas escalas e incluídos mais campos para obtenção de dados do consumidor.

A amostra desta pesquisa foi do tipo não probabilística, que, segundo Malhotra (2001), pode oferecer boas estimativas das características da população especificada na colocação do problema, embora Sampieri e outros (2006) sugiram que ela não é recomendada quando o objetivo é generalizar as conclusões do estudo. Os respondentes foram selecionados por conveniência (MALHOTRA, 2001; SAMPIERI e outros, 2006). Não foram oferecidos aos respondentes incentivos para participar.

Com base em estudos de campo anteriores (SPANGENBERG e outros, 1996; MATILLA e WIRTZ, 2001; MICHON e outros, 2005; PARSONS, 2009), foi definido que deveriam ser entrevistados, no mínimo, 110 respondentes por grupo, para que, após as eventuais 
eliminações, restasse uma quantidade próxima a 100. Para conseguir o máximo de equivalência entre os grupos, os dias para a coleta de dados (sete dias para cada grupo) foram planejados levando-se em consideração o calendário, para evitar coincidência com datas festivas e períodos promocionais no centro comercial (CHEBAT e MICHON, 2003). A coleta aconteceu entre julho e agosto de 2009.

\section{Pré-teste e calibração do aroma}

Entre os procedimentos essenciais em pesquisas sobre influência do estimulo olfativo em indivíduos, estão o pré-teste e a calibração do aroma a ser usado (EHRLICHMAN e HALPERN, 1988; SPANGENBERG e outros, 1996; CHEBAT e MICHON, 2003; SPANGENBERG e outros, 2005; BOSMANS, 2006; PARSONS, 2009). Para gerar comportamentos de aproximação dos consumidores, a fragrância escolhida deveria ser agradável (MEHRABIAN e RUSSELL, 1974; DONOVAN e ROSSITER, 1982; SPANGENBERG e outros, 1996; MILOTIC, 2003; PARSONS, 2009) e sua capacidade de ativação deveria estar entre estimulante e neutra (MEHRABIAN e RUSSELL, 1974; DONOVAN e ROSSITER, 1982; SPANGENBERG e outros, 1996). Outro fator primordial era a congruência entre cheiro e os elementos presentes na loja (MITCHELL e outros, 1995; SCHIFFERSTEIN e BLOCK, 2002; SPANGENBERG e outros, 2005; BOSMANS, 2006; PARSONS, 2009).

A escolha da fragrância envolveu o teste de cinco amostras, que foram borrifadas em uma bola de algodão colocada dentro de um frasco de vidro transparente. Seguindo a orientação de pesquisas anteriores (por exemplo, SPANGENBERG e outros, 1996; MORRIN e RATNESHWAR, 2000; SPANGENBERG e outros, 2005; BOSMANS, 2006; SPANGENBERG e outros, 2006), os respondentes, 20 no total, foram convidados a sentir as fragrâncias à medida que iam preenchendo o questionário de avaliação.

As avaliações da qualidade afetiva e da capacidade de ativação das fragrâncias foram inspiradas no procedimento adotado por Spangenberg e outros (1996), que usaram 10 pares de adjetivos bipolares: atraente/ não atraente, relaxante/tenso, confortável/desconfortável, positivo/negativo, estimulante/tedioso, bom/ruim, com vida/sem vida, claro/sombrio, motivante/desmotivante, interessante/ desinteressante. Os resultados revelaram que todas as amostras foram consideradas agradáveis e neutras em estimulação, ou seja, qualquer uma delas poderia ser escolhida.
Em relação à congruência, com base na temática da loja, foi solicitado às empresas de aromatização que enviassem fragrâncias que remetessem a praia, mar, maresia ou ao frescor da água. Para verificar a congruência com a loja, foi utilizada uma escala tipo Likert com quatro itens e sete pontos, inspirada na pesquisa de Spangenberg e outros (2005), cujos rótulos nas extremidades eram "discordo totalmente" e "concordo totalmente". Os quatro itens eram: "Esse aroma me traz a sensação de estar passeando na orla, sob coqueiros", "Esse aroma me lembra a brisa do mar", "Esse aroma captura o espírito de praia" e "Esse aroma seria apropriado para aromatizar uma loja desses produtos (fotos)". Conforme indicado no último item, foram apresentadas aos respondentes fotos dos produtos, tiradas na própria loja.

A amostra mais associada ao contexto praiano não poderia ser fornecida a tempo para atender ao cronograma do quase experimento e teve que ser descartada. Dessa forma, optou-se pela amostra Acqua, da empresa BioMist, que foi a segunda mais associada.

A calibração da intensidade do aroma escolhido foi feita com base na área da loja, seguindo a tabela do fornecedor da fragrância. A dispersão foi feita por meio de dois aparelhos dispenser B9, de funcionamento automático, regulados para dispersar $50 \mathrm{mg}$ a cada 2'02".

Algumas medidas foram tomadas no sentido de evitar a introdução de cheiros estranhos ao contexto de pesquisa (SPANGENBERG e outros, 2006). Assim, foi solicitado às vendedoras que não usassem perfumes durante os dias do quase experimento, que utilizassem produtos de limpeza neutros e que não borrifassem qualquer fragrância no ar.

\section{RESULTADOS}

Os dados coletados foram analisados quanto à normalidade, confiabilidade e dimensionalidade das escalas e, por fim, foi feito o teste de hipóteses. Os questionários foram previamente conferidos e eliminados aqueles que, durante a fase de coleta, não foram respondidos coerentemente, sendo 10 no total. As análises foram feitas com o auxílio de um pacote estatístico bastante utilizado nas ciências sociais.

Foram entrevistados 115 respondentes e obtidos 110 questionários válidos no grupo de controle, contra 112 entrevistados e 107 questionários válidos no 
grupo experimental. As amostras apresentam elevado grau de equivalência. Em ambas, houve proporção semelhante de homens e mulheres das classes A e B. Nos dois grupos, predominaram os casados, residentes na Região Metropolitana de Recife (RMR) e com curso superior. Os respondentes possuíam idade média semelhante, 45 anos, a maioria era cliente habitual da loja e apresentava frequências similares de visita e de compra.

Para verificar a adequação das técnicas planejadas para a análise dos dados, foi realizado o teste da normalidade das variáveis métricas para comparar com a distribuição normal, padrão de referência para métodos estatísticos. O teste de Kolmogorov-Smirnov (HAIR e outros, 2005) foi escolhido para calcular o nível de significância das diferenças das distribuições das escalas em relação a uma distribuição normal. Foram analisadas as escalas múltiplas de avaliação do ambiente (14 itens) e de loja (quatro itens), além das escalas simples de avaliação da variedade, qualidade e preço dos produtos, da intenção de retorno e da intensidade do sentimento em relação à loja. $O$ resultado do teste foi significativo $(\mathrm{p}<0,01)$ para todas as escalas, tanto para a amostra inteira quanto para cada grupo, indicando que todas elas são diferentes da distribuição normal. Dessa forma, a utilização de técnicas paramétricas não seria recomendada e optou-se, então, por técnicas não paramétricas ou de distribuição livre (NORUSIS, 1993; HAIR e outros, 2005).

Para garantir a confiabilidade dos dados - suscetibilidade ao erro de medida decorrente da utilização de variáveis múltiplas e sua combinação em técnicas multivariadas - foi obtido o coeficiente alfa de Cronbach, que mede a consistência interna de escalas múltiplas. Segundo Malhotra (2001) e Hair e outros (2005), coeficientes inferiores a 0,6 indicam confiabilidade insatisfatória da consistência interna. Esta pesquisa adotou duas escalas múltiplas para verificar a relação entre a presença/ausência de aroma ambiental e avaliação da loja (quatro itens) e do ambiente da loja (14 itens). Ambas apresentaram coeficientes satisfatórios no grupo de controle e no grupo experimental. Detalhes na Tabela 2.

Embora índices altos do alfa de Cronbach sugiram que a unidimensionalidade de uma escala múltipla existe, esse não é um teste definitivo para tal propósito. Em função disso, realizou-se análise fatorial, para avaliar a estrutura interna das correlações entre um grande número de variáveis. Segundo Hair e outros (2005), amostras com mais de 200 unidades podem obter significância com cargas fatoriais a partir de $\pm 0,400$. Nesta pesquisa, optou-se por considerar como significativas as cargas a partir de $\pm 0,500$.

A escala de avaliação da loja pelos consumidores apresentou um fator, ratificando a sua unidimensionalidade, com cargas entre 0,861 e 0,899 e variância explicada de $78,358 \%$. Na escala de avaliação do ambiente de loja, foi realizada uma primeira análise fatorial, que incluiu os 217 questionários válidos e apresentou três fatores, resultado não condizente com a sua proposta. Procedeu-se, então, a uma segunda tentativa, em que se optou, a priori, pela extração de dois fatores, que seriam afeto e ativação. Como no estudo de Spangenberg e outros (1996), não foi possível concluir que as duas dimensões extraídas expressavam os dois construtos de maneira clara. Dessa forma, optou-se por eliminar as três variáveis que estavam mais associadas ao segundo e terceiro fatores encontrados na primeira tentativa, para que se obtivesse apenas um fator, com uma variância explicada de 51,313\%. Segundo Hair e outros (2005), esse índice de variância é adequado para as ciências sociais, que são menos precisas. O coeficiente de confiabilidade, alfa de Cronbach, após a eliminação das três variáveis (confortável/desconfortável, relaxante/tenso e animado/desanimado) da escala de avaliação do ambiente de loja, foi de 0,8907, no total, e de 0,8832 e 0,8970, nos grupos de controle e experimental, respectivamente.

Ressalta-se que estudos anteriores que utilizaram essa escala, como Spangenberg e outros (2006), não procederam a análise fatorial ou não chegaram a resultado conclusivo sobre a sua unidimensionalidade. A tradução da escala representou uma dificuldade, uma vez que, para alguns termos, não havia uma denominação em português que representasse com precisão a ideia original em inglês. Isso pode ter contribuído para o não agrupamento das variáveis em um único fator.

A adequacidade (MSA) das amostras à aplicação da análise fatorial foi aferida pelo índice Kaiser-Meyer-Olkin (KMO), obtendo-se valores de 0,845 e 0,916 para as escalas de avaliação de loja e de avaliação do ambiente de loja, respectivamente. Segundo Malhotra (2001), valores KMO entre 0,5 e 1 indicam que a análise fatorial é adequada. Para avaliar o risco de as variáveis das escalas não serem correlacionadas na população, realizou-se o teste de Bartlett de esfericidade, no qual foram obtidos qui-quadrados de 561,908, com 6 graus de liberdade e significância p>0,01, e de 1.144,546, com 55 graus de liberdade e significância p >0,01, para as escalas de avaliação de loja e avaliação de ambiente de loja, respectivamente. 


\section{Tabela 1 - Coeficiente alfa de Cronbach}

\begin{tabular}{|c|c|c|c|c|c|}
\hline \multirow[b]{2}{*}{ Variáveis } & \multirow{2}{*}{$\begin{array}{c}\text { Carga } \\
\text { fatorial }\end{array}$} & \multirow{2}{*}{$\begin{array}{l}\text { Alfa de } \\
\text { Cronbach }\end{array}$} & \multicolumn{2}{|c|}{ Média (desvio-padrão) } & \multirow[b]{2}{*}{$\rho$} \\
\hline & & & $\begin{array}{l}\text { Grupo de } \\
\text { controle }\end{array}$ & $\begin{array}{c}\text { Grupo } \\
\text { experimental }\end{array}$ & \\
\hline Avaliação da loja & & 0,9014 & & & \\
\hline Favorável/desfavorável & 0,899 & & $6,51(0,81)$ & $6,42(0,86)$ & 0,348 \\
\hline Boa/ruim & 0,893 & & $6,69(0,65)$ & $6,53(0,68)$ & 0,035 \\
\hline Positiva/negativa & 0,887 & & $6,62(0,74)$ & $6,43(0,80)$ & 0,023 \\
\hline Moderna/antiquada & 0,861 & & $6,45(0,91)$ & $6,37(0,95)$ & 0,481 \\
\hline Aprovação da loja & & - & & & \\
\hline Rejeição/aprovação & - & & 12,05 & $12,19(2,03)$ & 0,737 \\
\hline Avaliação do ambiente de loja & & 0,8907 & & & \\
\hline Motivante/desmotivante & 0,815 & & $6,15(1,16)$ & $5,90(1,26)$ & 0,111 \\
\hline Interessante/desinteressante & 0,811 & & $6,38(0,90)$ & $6,24(0,92)$ & 0,169 \\
\hline Agradável/desagradável & 0,799 & & $6,55(0,87)$ & $6,37(1,00)$ & 0,08 \\
\hline Estimulante/tedioso & 0,78 & & $5,96(1,36)$ & $5,82(1,39)$ & 0,344 \\
\hline Bom/ruim & 0,774 & & $6,57(0,82)$ & $6,50(0,71)$ & 0,151 \\
\hline Positivo/negativo & 0,758 & & $6,51(0,82)$ & $6,37(0,84)$ & 0,116 \\
\hline Com vida/sem vida & 0,731 & & $5,97(1,32)$ & $5,83(1,31)$ & 0,266 \\
\hline Claro/sombrio & 0,63 & & $6,23(1,07)$ & $6,31(0,96)$ & 0,737 \\
\hline Colorido/desbotado & 0,618 & & $5,90(1,39)$ & $5,62(1,46)$ & 0,099 \\
\hline Atraente/não atraente & 0,57 & & $6,46(0,84)$ & $6,41(0,79)$ & 0,336 \\
\hline Aberto/fechado & 0,516 & & $5,82(1,42)$ & $5,62(1,22)$ & 0,061 \\
\hline Confortável/desconfortável & Excluída & & - & - & - \\
\hline Relaxante/tenso & Excluída & & - & - & - \\
\hline Animado/desanimado & Excluída & & - & - & - \\
\hline Avaliação dos produtos vendidos & & - & & & \\
\hline Variedade: adequada/inadequada & - & & $6,20(1,33)$ & $6,19(1,21)$ & 0,526 \\
\hline Preços: baixos/altos & - & & $4,66(1,25)$ & $4,59(1,32)$ & 0,721 \\
\hline Qualidade: alta/baixa & - & & $6,57(0,78)$ & $6,40(0,90)$ & 0,092 \\
\hline Possibilidade de retorno & & - & & & \\
\hline Possibilidade de retorno: alta/baixa & - & & $6,43(0,97)$ & $6,48(0,71)$ & 0,608 \\
\hline Permanência na loja & & - & & & \\
\hline Tempo médio de permanência & - & & $180,70 "$ & $210,67 "$ & 0,014 \\
\hline Distorção na percepção do tempo & & - & & & \\
\hline Tempo médio de permanência & - & & $180,70 "$ & $210,67 ”$ & 0,014 \\
\hline Tempo médio percebido & - & & $638,00 ”$ & $749,16 "$ & - \\
\hline Diferença tempos (distorção) & - & & $457,30 "$ & $538,49 "$ & 0,232 \\
\hline
\end{tabular}


A análise das médias, modas e medianas revelou que a loja foi muito bem avaliada pelos clientes tanto no tratamento de controle quanto no experimental, com a maior parte das médias acima de seis, como mostra a Tabela 2. As variáveis de avaliação de loja, avaliação do ambiente de loja, avaliação dos produtos ofertados (variedade e qualidade) e intenção de retorno à loja apresentaram modas e medianas iguais a sete nos dois tratamentos. O item preço, da variável avaliação dos produtos ofertados, cuja lógica é inversa, apresentou moda e mediana iguais a quatro nos dois tratamentos. As médias ligeiramente mais altas no grupo de controle também revelam que, para a quase totalidade das variáveis, não houve influência positiva na aromatização do ambiente. Para a comparação das médias dos grupos, foi especificado o nível de significância $\mathrm{p}<0,05$, bastante comum em pesquisas sobre aromatização no varejo (SCHIFFERSTEIN e BLOK, 2002; SPANGENBERG e outros, 2006; MCDONNELL, 2007; PARSONS, 2009).

Como a análise dos dados não poderia ser feita por meio de técnicas paramétricas, optou-se pelo teste U de Mann-Whitney, que é adequado para a comparação das médias de populações diferentes e para dados não paramétricos (NORUSIS, 1993; MALHOTRA, 2001). A técnica correspondente em análises paramétricas é o teste $t$.

A primeira hipótese focou a possibilidade de retorno do respondente para uma compra futura e apresentou média levemente maior no tratamento experimental, mas sem significância, como mostra a Tabela 2, o que revela que, contrariamente ao que concluíram Spangenberg e outros (1996) e Spangenberg e outros (2006), a presença de aroma ambiental não afetou a possibilidade de o consumidor retornar à loja. A hipótese H1 foi rejeitada.

Em relação à permanência na loja, os clientes do grupo experimental passaram quase 30 segundos a mais no local, em média, como mostra a Tabela 2 . Pode ser uma diferença pequena, mas é estatisticamente significativa, segundo o teste $U$ de Mann-Whitney. Esse tempo seria suficiente para o consumidor analisar mais um ou dois produtos, aumentando as chances de compra. Com isso, a hipótese H2 foi suportada, a presença de aroma ambiental aumentou o tempo de permanência dos consumidores na loja.

Para verificar a distorção na percepção do tempo gasto na loja, foram comparadas as diferenças entre os tempos médios reais de permanência no local e os tempos médios percebidos pelos respondentes em cada tratamento. A Tabela 2 mostra que, nos dois grupos, os consumidores perceberam ter gasto mais tempo na loja do que o real, mas com uma distorção um pouco mais acentuada no grupo experimental, como verificado por Spangenberg e outros (2006). Curiosamente, Spangenberg e outros (1996) identificaram um padrão oposto de distorção, em que os consumidores acharam ter permanecido menos tempo que o real em uma loja simulada e na presença de aroma ambiental. Entretanto, mesmo com o grupo experimental tendo apresentado maior distorção na percepção do tempo em relação ao grupo de controle, a diferença não foi estatisticamente significativa, o que corrobora a afirmação de Bone e Ellen (1999) de que há, na literatura, poucas evidências dos efeitos do aroma na distorção da percepção do tempo. Portanto, a hipótese H3 foi rejeitada, a presença de aroma ambiental não fez com que os clientes apresentassem distorção na percepção do tempo gasto na loja.

Como mencionado anteriormente, duas medições foram adotadas para a avaliação da loja. Na primeira, dos quatro itens utilizados em uma escala múltipla, dois foram estatisticamente significantes, boa/ruim e positiva/negativa, conforme mostra a Tabela 2. Embora a loja tenha sido bem-avaliada nos dois tratamentos, uma revisita às médias desses dois itens mostra que o grupo experimental fez uma avaliação um pouco mais baixa em relação ao grupo de controle. A segunda medição da variável avaliação de loja, aprovação da loja, mostrou que, em ambos os tratamentos, houve excelente avaliação, entretanto sem significância estatística entre os dois, como mostra a Tabela 2. Os resultados da análise item a item da primeira escala de avaliação de loja, juntamente com a análise da segunda escala, não fornecem base para concluir que as amostras pertencem a populações diferentes. Optou-se, em função do conjunto dos dados, pela rejeição da hipótese H4. Assim, a presença de aroma ambiental não produziu avaliações mais positivas da loja, como sugeriram os experimentos de laboratório de Spangenberg e outros (1996) e Parsons (2009), e os de campo de Chebat e Michon (2003) e Spangenberg e outros (2006).

Em relação à avaliação do ambiente de loja, procedeu-se à análise um a um de seus 11 itens, que não revelou diferenças significativas $(\mathrm{p}<0,05)$ em nenhum deles entre os dois tratamentos da pesquisa. Três itens, "aberto/fechado", "agradável/desagradável" e "colorido/desbotado", apresentaram significância marginal $(\mathrm{p}<0,1)$, como mostra a Tabela 2. Neles, o ambiente 
de loja sofreu uma avaliação levemente menor na presença do aroma. Por curiosidade, optou-se por avaliar os comportamentos dos três em gráficos de caixas (HAIR e outros, 2005), e a variável "colorido/desbotado" foi a única que revelou diferenças mais visíveis, sugerindo que os respondentes consideraram a loja mais colorida na ausência de aroma. É preciso lembrar que essa variável faz parte de uma escala múltipla e que a análise deve levar em consideração os vetores de médias sobre múltiplas variáveis dependentes ao longo dos grupos (HAIR e outros, 2005). Assim, devido à inacessibilidade a técnicas de análise multivariadas para dados não paramétricos e ao fato de que apenas a variável "colorido/desbotado" sugere uma diferença (negativa) entre os grupos, não há base para concluir que as amostras pertencem a populações diferentes, levando à rejeição da hipótese H5. A presença de aroma ambiental não produziu avaliações mais positivas do ambiente de loja.

A avaliação dos produtos ofertados empregou três escalas nas quais os respondentes avaliaram variedade, preços e qualidade. Como mostra a Tabela 2, não houve diferença estatisticamente significativa $(\mathrm{p}<0,05)$ na atitude dos respondentes dos dois grupos em relação aos itens julgados. Apenas a variável "qualidade" mostrou relevância marginal $(\mathrm{p}<0,1)$, com média menor no grupo experimental, mas uma análise em gráfico de caixa descartou qualquer diferença entre os tratamentos. Os resultados das três variáveis levaram à rejeição da hipótese H6. Assim, a presença de aroma ambiental não produziu avaliações mais positivas dos produtos vendidos na loja.

\section{DISCUSSÃO DOS RESULTADOS}

A presença de aroma ambiental não aumentou a possibilidade de o consumidor retornar à loja, hipótese $\mathrm{H} 1$, embora tenha resultado em maior tempo de permanência no local, hipótese H2. Segundo Bone e Ellen (1999), essa variável dependente parece ser a mais positivamente relacionada com a presença de aroma ambiental. Dois terços dos experimentos examinados por elas em um levantamento estatístico mostraram efeito semelhante. Pesquisas experimentais posteriores, como as de Morrin e Ratneshwar (2000) e as de Schifferstein e Blok (2002), também encontraram esse tipo de resposta.

As avaliações do consumidor em relação à loja, ambiente de loja e seus produtos, na presença de aroma ambiental, hipóteses H4, H5 e H6, também não repetiram os resultados positivos reportados em outros estudos nos quais essas variáveis dependentes foram medidas de modo semelhante, principalmente Spangenberg e outros (1996), Chebat e Michon (2003) e Spangenberg e outros (2006). Os dois últimos são estudos de campo. Em geral, os estudos de laboratório têm encontrado relações mais positivas entre aromatização no varejo e comportamentos de aproximação do consumidor. Em pesquisas dessa natureza, o controle da intensidade e dispersão do aroma é mais eficiente, assim como o isolamento de variáveis estranhas ao objeto de estudo. Além disso, os respondentes estão mais envolvidos na tarefa.

Um conjunto de fatores pode ter contribuído para a ausência de relações positivas entre a presença do aroma e as atitudes e comportamentos investigados. Um dos principais seria uma baixa congruência entre o aroma e a temática da loja. A aromatização da loja desta pesquisa foi feita com base nas características naturais dos produtos e na temática da atmosfera de loja, que remete a praia. Embora a congruência temática tenha apresentado resultados positivos em estudos como os de Spangenberg e outros (2006) e Parsons (2009), alguns autores fazem ressalvas à sua eficácia. Para Gulas e Bloch (1995), o aroma tem que levar em consideração todos os produtos do ambiente, e não apenas um, sob pena de prejudicar a avaliação dos outros, posição compartilhada por Spangenberg e outros (1996). Além disso, Schifferstein e Blok (2002) chamam a atenção para os riscos da congruência temática, em que o cheiro adotado pode trazer outros tipos de lembrança que não os pretendidos. O mesmo

\section{Tabela 2 - Resultados}

\begin{tabular}{|l|c|c|c|c|}
\hline \multicolumn{1}{|c|}{ Escala } & Itens & Grupo de controle & Grupo experimental & Total \\
\hline Avaliação da loja & 4 & 0,897 & 0,905 & 0,901 \\
\hline Avaliação do ambiente de loja & 14 & 0,876 & 0,902 & 0,890 \\
\hline
\end{tabular}


pode ter acontecido no contexto desta pesquisa. O fato de os consumidores terem demorado mais na loja aromatizada pode subsidiar essa suspeita, uma vez que não é necessário que o aroma seja congruente para retê-los por mais tempo, bastando que ele seja agradável (SPANGENBERG e outros, 1996).

É importante ressaltar que, apesar de algumas variáveis terem apresentado uma avaliação média inferior na presença de aroma, não há indícios de que o aroma tenha, de modo geral, piorado a atitude do consumidor em relação à loja. Isso permite inferir que o cheiro adotado não foi incongruente nem desagradável (MITCHELL e outros, 1995; D’ASTOUS, 2000; SPANGENBERG e outros, 2005).

Outro ponto que pode ter influenciado os resultados refere-se a problemas com a intensidade do cheiro na loja, que pode não ter sido a ideal. Esse aspecto é relevante porque, segundo Spangenberg e outros (2006), a intensidade do cheiro é muito importante na geração de comportamentos de aproximação. É necessário que a presença do aroma ambiental altere o estado de humor do consumidor de maneira positiva, para que isso se transfira para a avaliação de loja e produtos (BITNER, 1992; MITCHELL e outros, 1995; SPANGENGERG e outros, 1996; CHEBAT e MICHON, 2003). Houve muito cuidado na calibração da intensidade do aroma ambiental, uma vez que estímulos muito fortes são capazes de gerar comportamentos de afastamento (MEHRABIAN e RUSSELL, 1974; DONOVAN e ROSSITER, 1982) e, por se tratar de varejo de alimentos, especulou-se que a sensibilidade à intensidade poderia ser maior do que em varejos de outra natureza.

Pode ter contribuído para possíveis deficiências na difusão do aroma, afetando sua intensidade e homogeneidade no ambiente, o fluxo do ar-condicionado, projetado de modo a criar um corredor de ar no centro da loja, para não afastar a camada de ar frio sobre os expositores frigoríficos posicionados nas laterais, o que poderia comprometer a conservação dos produtos. Questões estéticas e de segurança também impediram uma melhor localização dos dispensers, embora eles não tenham ficado mal-localizados.

Por fim, uma das conclusões de Spangenberg e outros (1996) sugere que a presença de aroma ambiental dificilmente vai melhorar as avaliações de algo que já é bem-avaliado na ausência de aroma, como os produtos, o ambiente de loja e a própria loja. Segundo Isen e Shalker (1982), uma mudança no estado afetivo de uma pessoa pode fazer com que ela avalie positivamente estímulos anteriormente avaliados como negativos ou ambíguos, mas não vai afetar a atitude em relação àqueles já considerados positivos.

A utilização de aroma ambiental em lojas tem como principal mecanismo de ação justamente provocar alterações nos sentimentos das pessoas, no humor (GARDNER, 1985; MITCHELL e outros, 1995), para que esse novo estado de afeto, positivo, se transfira para o ambiente, incluindo os produtos (GARDNER, 1985; BITNER, 1992; MITCHELL e outros, 1995; SPANGENBERG e outros, 1996; CHEBAT e MICHON, 2003). Se loja e produtos já são avaliados positivamente, é menos provável que a presença de aroma vá exercer impacto significante nessas avaliações. A loja e os produtos pesquisados foram muito bem avaliados pelos consumidores no tratamento de controle como mostrado anteriormente, levando a crer que, embora não fosse impossível, seria bastante improvável que a aromatização ambiental conduzisse a uma melhora estatisticamente significativa da atitude em relação à loja e seus produtos.

Em resumo, as análises revelam que a presença de aroma ambiental em um varejo real não foi capaz de afetar positivamente as avaliações de loja, ambiente de loja e produtos, o que pode ser explicado por fatores como a congruência do aroma e sua intensidade no ambiente, que não atingiram uma condição ideal, ou pelo fato de a loja ter sido muito bem avaliada na ausência de aroma, restando pouco a ser feito pelo estímulo olfativo. Nesse estudo, a presença de aroma não foi capaz de aumentar a intenção de retorno, mas foi capaz de reter o consumidor por mais tempo na loja.

\section{CONSIDERAÇÕES FINAIS}

A revisão teórica mostrou que não há um consenso sobre a influência do estímulo olfativo. Embora muitos pesquisadores tenham encontrado relações positivas com os comportamentos de aproximação do consumidor no ponto de venda, é preciso reconhecer que há uma considerável quantidade de estudos não conclusivos, principalmente os de campo.

Em sua revisão de artigos sobre aromatização no varejo, Bone e Ellen (1999) verificaram que 54\% daqueles relacionados a avaliações do consumidor apresentaram resultados estatisticamente insignificantes. Desde então, outros estudos foram realizados com 
novas técnicas e abordagens de pesquisas e resultados não significantes continuaram a aparecer, como o de Spangenberg e outros (2005), sobre avaliações de loja, ambiente e produtos, realizado em laboratório, e o de Parsons (2009), que falhou ao reproduzir no campo seu bem-sucedido experimento de laboratório sobre avaliação do ambiente de loja na presença de aroma associado.

Entretanto, não se pode ignorar os indícios de influência positiva, como a maior permanência na loja, observada em vários estudos. Durante o tratamento experimental desta pesquisa, muitos clientes comentaram o agradável aroma que estavam sentindo na loja, alguns até com espanto, mas isso não parece ter sido capaz de melhorar julgamentos que já eram bastante satisfatórios. Esses comentários levam a crer que outras metodologias podem ser utilizadas para capturar a natureza positiva do fenômeno em outra perspectiva.

Em alguns tipos de varejo, onde os produtos comercializados não possuem uma característica olfativa específica, ou quando ela é eliminada, como no caso estudado, é possível que bons resultados só sejam atingidos com o desenvolvimento de aromas específicos, que levem em consideração as características próprias da loja e as sensações e comportamentos desejados pelo varejista. Isso significa que a utilização de aromas genéricos de catálogo, como o que foi usado nesta pesquisa (em função de prazo e custos), não parece ser a opção mais efetiva.

Do ponto de vista prático, este estudo contribui para alertar os varejistas interessados em aromatizar suas lojas sobre a complexidade do processo e os riscos que uma escolha malfeita de fragrância pode trazer ao negócio. Fica evidente que a decisão sobre aromatização não pode ser algo intuitivo ou sujeito a preferências pessoais. É preciso levar em consideração todos os atores envolvidos no processo, a proposta do negócio e os resultados esperados.

A principal contribuição acadêmica do estudo foi testar, no campo, conceitos que têm sido mais eficazes no laboratório, mostrando que o tema continua inconclusivo e que mais pesquisas são necessárias. Além disso, embora os resultados não tenham sido positivos, há indícios, no comportamento das pessoas, de que há influência favorável do aroma e que constatar isso cientificamente pode ser uma questão de ajuste de metodologia. A revisão teórica, por exemplo, não encontrou estudos qualitativos sobre o tema, sendo que muitos respondentes se reportaram de maneira qualitativa (positiva) ao aroma presente na loja.
Por fim, sugere-se que estudos futuros investiguem a influência do aroma na prestação de serviços, principalmente na interação com a força de vendas. Além disso, tanto no varejo quanto em serviços, é relevante estudar a influência do aroma na produtividade e satisfação da própria força de vendas, que, ao contrário dos clientes, está sob a influência do aroma permanentemente. Durante a realização deste estudo, ficou claro que algumas vendedoras da loja pesquisada aprovaram a utilização do aroma, enquanto outras revelaram estar incomodadas. São também bem-vindos os estudos que combinem aroma e outros estímulos ambientais.

\section{REFERÊNCIAS}

BELLIZZI, J. A; CROWLEY, A. E; HASTY, R. W. The effects of color in store design. Journal of Retailing, v. 59, n. 1, p. 21-45, 1983.

BITNER, M. J. Servicescapes: the impact of physical surroundings on customers and employees. Journal of Marketing, v. 56, n. 2, p. 57-71, 1992.

BONE, P. F; ELLEN, P. S. Scents in the marketplace: explaining fraction of olfaction. Journal of Retailing, v. 75, n. 2, p. 243-262, 1999.

BONE, P. F; JANTRANIA, S. Olfaction as a cue for product quality. Marketing Letters, v. 3, n. 3, p. 289-296, 1992.

BOSMANS, A. Scents and sensibility: when do (in)congruent ambient scents influence product evaluations? Journal of Marketing, v. 70, n. 3, p. 32-43, 2006.

CHEBAT, J; MICHON, R. Impact of ambient odors on mall shoppers' emotions, cognition, and spending. A test of competitive causal theories. Journal of Business Research, v. 56, n. 7, p. 529-539, 2003.

CORBETT, M. Scents of identity: organisation studies and the cultural conundrum of the nose. Culture and Organization, v. 12, n. 3, p. 221-232, 2006.

D'ASTOUS; A. Irritating aspects of the shopping environment. Journal of Business Research, v. 49, n. 2, p. 149156, 2000. 
DAVIES, B. J; KOOIJMAN, D; WARD, P. The sweet smell of success: olfaction in retailing. Journal of Marketing Management, v. 19, n. 5-6, p. 611-627, 2003.

DAVIDOFF; L. Introdução à psicologia. 3. ed. São Paulo: Pearson, 2001.

DONOVAN, R. J; ROSSITER, J. R. Store atmosphere: an environmental psychology approach. Journal of Retailing, $\mathrm{v}$. 58, n. 1, p. 34-57, 1982.

DOTY, R. L. Studies of human olfaction from the University of Pennsylvania Smell and Taste Center. Chemical Senses, v. 22, n. 5, p. 565-586, 1997.

DUBÉ, L; MORIN, S. Background music pleasure and store evaluation: intensity effects and psychological mechanisms. Journal of Business Research, v. 54, n. 2, p. 107-113, 2001.

EHRLICHMAN, H; HALPERN, J. N. Affect and memory: effects of pleasant and unpleasant odors on retrieval of happy an unhappy memories. Journal of Personality and Social Psychology, v. 55, n. 5, p. 769-779, 1988.

GARDNER, M. P. Mood states and consumer behavior: a critical review. Journal of Consumer Research, v. 12, n. 3, p. 281-300, 1985.

GOLDKUHL, L; STYVÉN, M. Sensing the scent of service success. European Journal of Marketing, v. 41, n. 11-12, p. 1297-1305, 2007.

GULAS, C. S; BLOCH, P. H. Right under our noses: ambient scent and consumer responses. Journal of Business Psycho$\log y$, v. 10, n. 1, p. 87-98, 1995.

HAIR, J. F; ANDERSON, R. E; TATHAM, R. L; BLACK, W. C. Análise multivariada de dados. 5. ed. Porto Alegre: Bookman, 2005.

HERZ, R. S; ENGEN, T. Odor memory: review and analysis. Psychonomic Bulletin E Review, v. 3, n. 3, p. 300-313, 1996.

HIRSCHMAN, E. C; HOLBROOK, M. B. Hedonic consumption: emerging concepts, methods and propositions. Journal of Marketing; v. 46, n. 3, p. 92-101, 1982.

HOLBROOK, M. B; HIRSCHMAN, E. C. The experiential aspects of consumption: consumer fantasies, feelings, and fun. Journal of Consumer Research, v. 9, n. 2, p. 132-140, 1982.
ISEN, A. M; SHALKER, T. E. The effect of feeling state on evaluation of positive, neutral, and negative stimuli: when you "accentuate the positive" do you "eliminate the negative”? Social Psychology Quarterly, v. 45, n. 1, p. 58-63, 1982.

KOTLER, P. Atmospherics as a marketing tool. Journal of Retailing, v. 4, n. 49, p. 48-64, 1973.

MALHOTRA, N. Pesquisa de marketing: uma orientação aplicada. 3. ed. Porto Alegre: Bookman, 2001.

MATILLA, A. S; WIRTZ, J. Congruency of scent and music as a driver of in-store evaluations and behavior. Journal of Retailing, v. 77, n. 2, p. 273-289, 2001.

McDONNELL, J. Music, scent and time preferences for waiting lines. International Journal of Bank Marketing, v. 25, n. 4, p. 223-237, 2007.

MEHRABIAN, A; RUSSELL, J. A. An approach to environmental psychology. Cambridge: MIT Press, 1974.

MICHON, R; CHEBAT, J; TURLEY, L. W. Mall atmospherics: the interaction effects of the mall environment on shopping behavior. Journal of Business Research, v. 58, n. 5, p. 576-583, 2005.

MILLIMAN, R. Using background music to affect the behavior of supermarket shoppers. Journal of Marketing, v. 46, n. 3, p. 86-91, 1982

MILOTIC, D. The impact of fragrance on consumer choice. Journal of Consumer Behaviour. v. 3, n. 2, p. 179-191, 2003.

MITCHELL, D. J; KAHN, B. E; KNASKO, S. C. There's something in the air: effects of congruent or incongruent ambient odor on consumer decision making. Journal of Consumer Research, v. 22, n. 2, p. 229-238, 1995.

MORRIN, M; RATNESHWAR, S. The impact of ambient scent on evaluation, attention, and memory for familiar and unfamiliar brands. Journal of Business Research, v. 49, n. 2, p. $157-165,2000$.

NORUSIS, M. J. SPSS ${ }^{\circledR}$ for Windows ${ }^{T M}$ : Base System User's Guide, Release 6.0. Chicago: SPSS Inc., 1993.

PAL, J. W; BYROM, J. W. The five Ss of retail operations: a model and tool for improvement. International Journal of Retail E Distribution Management, v. 31, n. 10, p. 518-528, 2003. 
PARENTE, J. Varejo no Brasil: gestão e estratégia. São Paulo: Atlas, 2000.

PARSONS, A. G. Use of scent in a naturally odourless store. International Journal of Retail \& Distribution Management, v. 37, n. 5, p. 440-452, 2009.

RICHARDSON, J. T. E; ZUCCO, G. M. Cognition and olfaction: a review. Psychological Bulletin, v. 105, n. 3, p. 352-360, 1989.

SCHIFFERSTEIN, H. N. J; BLOK, S. T. The signal function of thematically (in)congruent ambient scents in a retail environment. Chemical Senses, v. 27, n. 6, p. 539-549, 2002.

SCHINDLER, R. M; HOLBROOK, M. B. Nostalgia for early experience as a determinant of consumer preferences. Psychology \& Marketing, v. 20, n. 4, p. 275-302, 2003.

SCHMITT, B. Experiential marketing. Journal of Marketing Management, v. 15, n. 1, p. 53-67, 1999.

SAMPIERI, R. H.; COLLADO, C. F.; LUCIO, P. B. Metodologia de Pesquisa. 3. ed. São Paulo: McGraw-Hill, 2006.

SPANGENBERG, E. R; CROWLEY, A. E; HENDERSON, P. Improving the store environment: do olfactory cues affect evaluations and behaviours? Journal of Marketing, v. 60, n. 2, p. 67-80, 1996.

SPANGENBERG, E. R; GROHMANN, B; SPROTT, D. E. It's beginning to smell (and sound) a lot like Christmas: the interactive effects of ambient scent and music in a retail setting. Journal of Business Research, v. 58, n. 11, p. 15831589, 2005.

SPANGENBERG, E. R; SPROTT, D. E; GROHMANN, B; TRACY, D. L. Gender-congruent ambient scent influences on approach and avoidance behaviors in a retail store. Journal of Business Research, v. 59, n. 12, p. 1281-1287, 2006.

STROEBELE, N; DE CASTRO, J. M. Effect of ambience on food intake and food choice. Nutrition, v. 20, n. 9, p. 821-838, 2004.

TURLEY, L.W; CHEBAT, J. C. Linking retail strategy, atmospheric design and shopping behaviour. Journal of Marketing Management, v. 18, n. 1/2, p. 125-144, 2002.
TURLEY, L.W; MILLIMAN, R. E. Atmospheric effects on shopping behavior: a review of the experimental evidence. Journal of Business Research, v. 49, n. 2, p. 193-211, 2000.

WAKEFIELD, K. L; BAKER, J. Excitement at the mall: determinants and effects on shopping response. Journal of Retailing, v. 74, n. 4, p. 515-539, 1998. 